\title{
Numerical parametric study of the evaporation rate of a liquid under a shear gas flow: experimental validation and the importance of confinement on the convection cells and the evaporation rate
}

\author{
H. Machrafi ${ }^{1}$, Y. Lyulin ${ }^{2,3}$, C.S. Iorio ${ }^{4}$, O. Kabov ${ }^{2}$, P.C. Dauby ${ }^{1}$ \\ ${ }^{1}$ Université de Liège, Institut de Physique B5a, Allée du 6 Août 19, B-4000 Liège 1, Belgium \\ ${ }^{2}$ Institute of Thermophysics, Russian Academy of Sciences, Prosp. Lavrentyev 1, Novosibirsk \\ 630090, Russia \\ ${ }^{3}$ Novosibirsk State University, Pirogova Str. 2, Novosibirsk, 630090, Russia \\ ${ }^{4}$ Université libre de Bruxelles, Microgravity Research Center, Ecole Polytechnique, \\ CP165/62, avenue F.D. Roosevelt, 50, 1050 Brussels, Belgium
}

\begin{abstract}
Evaporation can cause instability due to cooling effects on the density and surface tension. This causes, respectively, Rayleigh and Marangoni instabilities. When these instabilities grow sufficiently, selfsustained convection occurs. This convection causes changes into the evaporation rate and heat transfer rate. This also could change the heat transfer via the evaporation rate and can be important for industrial applications. It is the purpose of this paper to investigate the relation that exists between the overall evaporation rate and a set of parameters: temperature, gas flow and liquid thickness. Three-dimensional numerical simulations have been performed for this purpose and the results have been validated by means of an experimental setup, on which the numerical geometry has been based, characterized by a liquid evaporating through an opening in a cover sheet under a shear gas flow. It is shown that the temperature and the gas flow increase the evaporation rate. More interestingly, a maximum is observable for the evaporation rate as function of the liquid thickness. The explanation for these phenomena are drawn from the 3D numerical simulations. It appears that the maximum evaporation rate as a function of the liquid thickness depends on the confinement of the convection cells by the cover sheet, being assisted by the gas flow.
\end{abstract}

Keywords: Evaporation, Shear flow, Confinement, Convection cell, Numerical modelling, Experimental validation

\section{Introduction}

Rayleigh-Bénard (RB), buoyancy-driven, and Marangoni-Bénard (MB), surface-tensiondriven, convection of a fluid heated from below and/or cooled from above is a classical problem in fluid dynamics (e.g. Pearson, 1958; Bénard, 1901; Norman et al., 1977; Colinet et al., 2001). It played a crucial role in the development of stability theory in hydrodynamics (Chandrasekhar, 1981; Drazin and Reid, 1981) and had been paradigmatic in pattern formation and in the study of spatial-temporal chaos (Getling, 1998; Bodenschatz et al., 2000). RB and MB convection also play important roles in recent stability analyses where evaporation is the driving force (Haut and Colinet, 2005; Bestehorn, 2007; Machrafi et al., 2011; Machrafi et al., 2013a). From an applied viewpoint, thermally driven flows are of utmost importance. A non-exhaustive list of examples comprises thermal convection in the atmosphere, in oceans, buildings, process technology. These thermal flows can give rise to certain self-sustained patterns that can be stationary or can change into other patterns or even to chaotic structures. We are here especially interested in evaporation-related thermal flows. These have been studied theoretically, numerically and experimentally, e.g. Bestehorn and Merkt (2006), Iorio et al. (2011), Lyulin and Kabov (2014) and Machrafi et al. (2014), to mention a few. This work falls into a general framework which consists of observing the behavior of the evaporation rates as a function of a set of parameters: the temperature, the gas flow and the liquid thickness. In previous work, such a configuration has been studied with regard to theoretical instability thresholds (Machrafi et al., 2013b). What is of interest here, is a three-dimensional numerical simulation of the 
temperature and fluid motion in the liquid for a liquid evaporating into a horizontal nitrogen gas flow. The chosen liquid is HFE7100 (an electronics coolant liquid produced by $3 \mathrm{M}$ ). The numerical simulations (CFD) are performed using the software ComSol (finite elements method). The numerical results are validated experimentally, using an experimental setup (Lyulin and Kabov, 2014), on which the numerical simulation geometry is based. The evaporation, taking place at the upper surface of the liquid layer results into the cooling of the liquid surface. This cooling induces temperature gradients in the liquid. These temperature gradients are in turn responsible for the development of a thermal boundary layer that develops from the liquid-gas interface into the bulk liquid. Since density and surface tension depend both on the temperature, the temperature gradients cause them to change. As a consequence, locally colder zones of fluid will have a higher density and surface tension than their neighboring fluid particles, which will cause the fluid to move. When the thermal gradient exceeds a certain threshold, instability sets in and self-sustained convective patterns take place. As time proceeds, these patterns can become chaotic. The numerical setup in the present work is based on a socalled evaporator, which takes part of a pre-flight analysis of the EVAPORATION PATTERNS (EP) (previously named CIMEX) experimental setup of the European Space Agency (ESA), to be flown aboard a satellite in the near future. A sketch of the physical system is presented in Fig. 1.

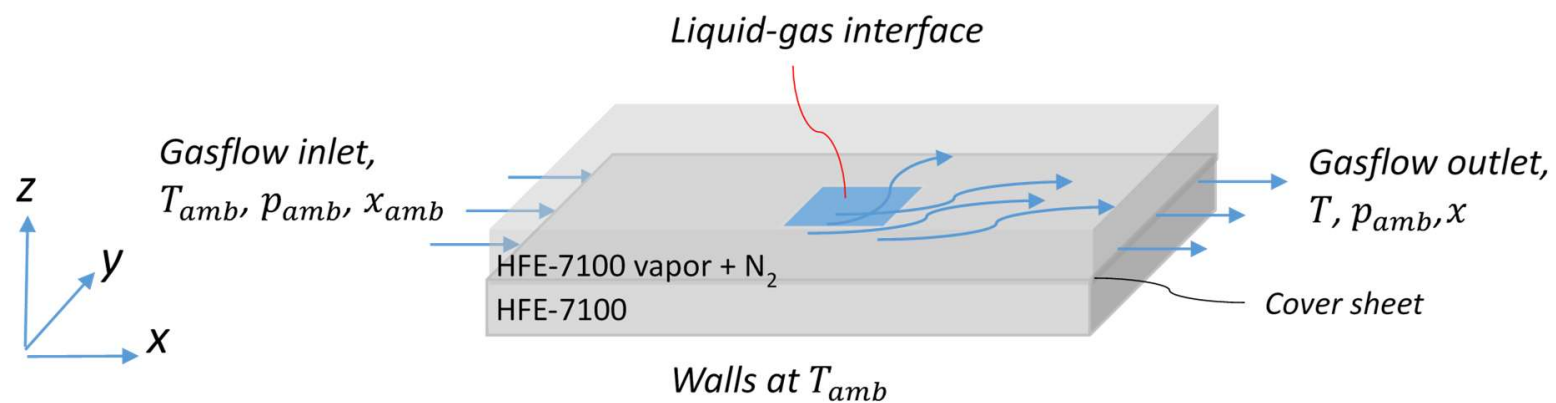

FIG. 1. The schematic of the physical system

The physical setup that will be numerically studied has overall dimensions of $50 \mathrm{~mm}$ by $50 \mathrm{~mm}$ with a certain liquid height, $h_{\text {liq }}$, variable from $1.5 \mathrm{~mm}$ to $8 \mathrm{~mm}$. The evaporated liquid is considered to be constantly replenished from the bottom, so that the gas-liquid interface, present within an opening $10.6 \mathrm{~mm}$ by $10.6 \mathrm{~mm}$, remains at the same level. Evaporation is allowed through this opening, while gas absorption into the liquid is neglected. Above it, there is a gas channel with a height, $h_{\text {gas }}$, of $5 \mathrm{~mm}$ and a width of $50 \mathrm{~mm}$. In this channel, nitrogen is injected with flow rates, $Q_{\text {gas }}$, between 100 and $5000 \mathrm{ml} / \mathrm{min}$. The gas flow at the gas channel entrance has a certain imposed relative humidity $\eta$ in HFE-7100 vapor in nitrogen. The relative humidity can be varied from $0 \%$ to $100 \%$, expressed in an ambient (denoted by the subscript "amb") molar fraction $x_{a m b}$ via the relation $x_{a m b} \equiv \eta p_{\text {sat }}\left(T_{a m b}\right) / p_{a m b}$, where $p_{\text {sat }}$ is the saturation pressure of the vapor, while $T_{a m b}$ is an ambient temperature between 20 and $40{ }^{\circ} \mathrm{C}$ and $p_{a m b}$ an ambient pressure of $1 \mathrm{~atm}$. At the exit, we have an open boundary with a constant pressure, where the molar fraction satisfies a zero-flux condition (meaning that we assume that after the exit the molar fraction does not change, which is approximately the case in the EP experiment). Between the gas and liquid layers a cover sheet is placed to separate them, except for an assumed undeformable $10.6 \mathrm{~mm}$-square opening. The rest of the interfaces of the system are walls kept at a constant temperature $T_{a m b}$. One of the goals of this EP experiment is to quantify mass-transfer processes across interfaces, i.e. evaporation, and their coupling with surfacetension-driven (Marangoni) and density-driven (Rayleigh) flows/convection in a liquid. In this 
work, we focus on presenting a parametric study of such convection, in order to analyze specific behaviour of the evaporation process as a function of those parameters. Particular attention is given to the effect of the liquid layer thickness, which takes into consideration the effect confinement by the cover can have on the number of convection cells and its result on the evaporation rate. The paper is organized as follows. Section 2 provides a mathematical description of the model with the boundary conditions. Section 3 deals with the numerical part as well as the experimental setup used for validating the numerical results. Section 4 shows the results, followed by the conclusions in Section 5.

\section{Model}

The Boussinesq approximation is adopted for both phases of the system, implying that the material properties of the fluids are treated as constant except for the density in the buoyancy terms, where it depends linearly on the temperature and the molar fraction, and the surface tension in the stress balance at the interface, where it depends linearly on the temperature. Before starting with the equations in the bulk and the boundary conditions, we provide the expressions for the density as used in the buoyancy terms (under the Boussinesq approximation):

$$
\begin{aligned}
& \tilde{\rho}_{l}=\rho_{l}\left(1-\alpha_{l}\left(T_{l}-T_{l, 0}\right)\right) \\
& \tilde{\rho}_{g}=\rho_{g}\left(1-\alpha_{g}\left(T_{g}-T_{g, 0}\right)-\varepsilon_{g}\left(\chi_{g}-\chi_{g, 0}\right)\right)
\end{aligned}
$$

In (1) and (2), $\rho$ is the density (the tilde denotes the density under the Boussinesq approximation), $\alpha$ and $\varepsilon$ denote the thermal and the solutal expansion coefficients. The subscripts "l" and " $g$ " relate to the liquid and gas phases, respectively. The subscript " 0 " refers to a state with a certain typical value of the temperatures and molar fraction, here taken to be $T_{l, 0}=T_{g, 0}=T_{a m b}$ and $\chi_{g, 0}=\chi_{a m b}$. The densities $\rho_{l}$ and $\rho_{g}$ are taken at ambient conditions. The equations for the bulk, in both the gas and liquid phases, are then given by

$\nabla \cdot \vec{v}_{l}=0$

$\rho_{l} \frac{\partial \vec{v}_{l}}{\partial t}=-\rho_{l}\left(\vec{v}_{l} \cdot \vec{\nabla}\right) \vec{v}_{l}-\vec{\nabla} p_{l}+\mu_{l} \nabla^{2} \vec{v}_{l}+\rho_{l}\left(1-\alpha_{l}\left(T_{l}-T_{l, 0}\right)\right) \vec{g}$,

$\frac{\partial T_{l}}{\partial t}=-\left(\vec{v}_{l} \cdot \vec{\nabla}\right) T_{l}+\kappa_{l} \nabla^{2} T_{l}$,

$\nabla \cdot \vec{v}_{g}=0$

$\rho_{g} \frac{\partial \vec{v}_{g}}{\partial t}=-\rho_{g}\left(\vec{v}_{g} \cdot \vec{\nabla}\right) \vec{v}_{g}-\vec{\nabla} p_{g}+\mu_{g} \nabla^{2} \vec{v}_{g}+\rho_{g}\left(1-\alpha_{g}\left(T_{g}-T_{g, 0}\right)-\varepsilon_{g}\left(\chi_{g}-\chi_{g, 0}\right)\right) \vec{g}$,

$\frac{\partial T_{g}}{\partial t}=-\left(\vec{v}_{g} \cdot \vec{\nabla}\right) T_{g}+\kappa_{g} \nabla^{2} T_{g}$

$\frac{\partial \chi_{g}}{\partial t}=-\left(\vec{v}_{g} \cdot \vec{\nabla}\right) \chi_{g}+D_{g} \nabla^{2} \chi_{g}$

where $\vec{v}$ and $p$ are the (barycentric) velocity and pressure fields, respectively. Furthermore, $D_{g}$ is the diffusion coefficient of HFE vapor in $\mathrm{N}_{2}$ and $\mu$ the dynamic viscosity. Note that the thermal diffusivity $\kappa$ is also defined by $\kappa=\lambda \rho^{-1} c_{p}^{-1}$, with $\lambda$ and $c_{p}$ being the thermal conductivity and heat capacity, respectively. For the liquid and gas phases respectively, (3) and 
(6) are the continuity equations for incompressible fluids, (4) and (7) are the momentum equations, (5) and (8) express the energy conservation, whereas (9) stands for the species conservation in the gas phase. The last terms of Eqs. (4) and (7), respectively, represent the density-driven volume forces (Rayleigh effect). Note that due to the high molecular weight of HFE-7100, considering a constant gas mixture density in all but the gravitational term can be questionable. To validate this simplifying assumption, we have numerically checked that as far as the evaporation rate is concerned, the effect of the density variations related to the vapour concentration is less than $2 \%$. Because of the smallness of this deviation, and also in order to keep a reasonable computing time (being drastically increased when a non-constant density is taken into account), we have disregarded this effect in our approach.

The following boundary conditions are used at the bottom and vertical walls, surrounding the liquid:

$\vec{v}_{l}=0, \quad T_{l}=T_{a m b}$

At the top of the gas channel and the lateral walls (parallel to the direction of the gas flow), we have

$T_{g}=T_{a m b}, \quad-\left(\vec{\nabla} x_{g} \cdot \vec{n}\right)=0, \quad \vec{v}_{g}=0$

where $\vec{n}$ is the normal vector. The symbols $u, v$ and $w$ stand for the $x, y$ and $z$ velocity field components, respectively. The entrance of the gas channel is described by

$\left(\vec{v}_{g} \cdot \vec{e}_{x}\right)=Q_{\text {flow }} / A_{g, \text { in }}, \quad T_{g}=T_{a m b}, \quad x_{g}=x_{a m b}$

where $\vec{e}_{x}$ is the horizontal vector in gas flow direction, $Q_{\text {flow }}$ the volumetric gas flow and $A_{g \text {,in }}$ the surface of the entrance. The exit of the gas channel is described as an outflow with an external imposed (known) pressure:

$p_{g}=p_{a m b}$

A cover sheet (made out of solid stainless steel with a thickness of $200 \mu \mathrm{m}$ ) that is between the liquid and the gas is modelled by heat conduction in a solid, with zero velocity and temperature continuity as boundary conditions at both liquid and gas sides. Consider now the conditions at the liquid-gas interface. First, the continuity of the temperature and of the tangential velocity components is assumed:

$T_{l}=T_{g}, \quad u_{l}=u_{g}, \quad v_{l}=v_{g}$

The energy conservation at the interface is described by a balance between the heat fluxes arriving from both phases and the heat of evaporation, which reads

$-\lambda_{l} \frac{\partial T_{l}}{\partial z}+\lambda_{g} \frac{\partial T_{g}}{\partial z}=J L$

where $L$ is the latent heat of evaporation, whereas $J$ is the evaporation flux. The evaporation flux is expressed (Machrafi et al., 2013b) as a function of the molar fraction in the gas phase as

$J=-\frac{n_{g} D_{g} M}{1-x_{g}} \frac{\partial x_{g}}{\partial z}$ 
where $M$ is the molar mass of HFE-7100 and $n_{g}$ is the molar gas density $n_{g}=p_{a m b} /\left(R T_{a m b}\right)$, with $R$ the universal gas constant. In expressing the tangential stress balance at the interface, it is assumed that the surface tension $\gamma$ between the liquid and the gas depends on the temperature in the liquid. Similarly to (1) and (2), one can write

$\gamma=\gamma_{0}-\gamma_{T}\left(T_{l}-T_{l, 0}\right)$

with $\gamma_{0}$ the surface tension at temperature $T_{l, 0}$ and $\gamma_{T}=-\partial \gamma / \partial T$. Then, the conditions expressing the tangential stress balance (Marangoni effect) at the interface are

$-\mu_{g}\left(\frac{\partial w_{g}}{\partial x}+\frac{\partial u_{g}}{\partial z}\right)+\mu_{l}\left(\frac{\partial w_{l}}{\partial x}+\frac{\partial u_{l}}{\partial z}\right)+\gamma_{T} \frac{\partial T_{l}}{\partial x}=0$,
$-\mu_{g}\left(\frac{\partial w_{g}}{\partial y}+\frac{\partial v_{g}}{\partial z}\right)+\mu_{l}\left(\frac{\partial w_{l}}{\partial y}+\frac{\partial v_{l}}{\partial z}\right)+\gamma_{T} \frac{\partial T_{l}}{\partial y}=0$,

A local equilibrium hypothesis at the surface is adopted. The evaporation process for the HFE7100 vapor is governed by the Clausius-Clapeyron relation for an ideal gas

$x_{g \Sigma}=\frac{p_{s a t}\left(T_{\Sigma}\right)}{p_{a m b}}=\frac{p_{s a t}\left(T_{a m b}\right)}{p_{a m b}} e^{\left(-\frac{L M}{R}\left(\frac{1}{T_{\Sigma}}-\frac{1}{T_{a m b}}\right)\right)}$,

where the symbol " $\Sigma$ " denotes the liquid-gas interface.

Initially, the liquid is at rest $\left(\vec{v}_{l}=0\right)$, with an initial temperature $T_{l}=T_{a m b}$. The gas has an initial temperature $T_{g}=T_{a m b}$ and initial vapor molar fraction $x_{g}=x_{a m b}$. In this work, the ambient temperature is a parameter, whilst the ambient pressure $p_{a m b}=1$ atm. The ambient molar fraction in the gas phase $x_{a m b}$ is taken to be zero in this work, i.e. we don't consider any humidity in the gas phase. The temperature-dependence of the properties are neglected, except for the saturation pressure, following the dependence described by Eq. (20). The physical properties used in this paper can be found in Table 1. The references to the sources of these physical properties can be found in previous work (Machrafi et al., 2011).

Table 1. Physical properties of the HFE-7100 system.

\begin{tabular}{llcc}
\hline \hline Symbol & Physical property & Value in the liquid phase & Value in the gas phase \\
\hline \hline$\rho\left[\mathrm{kg} / \mathrm{m}^{3}\right]$ & Density & $1.482^{*} 10^{3}$ & 1.145 \\
$c_{p}[\mathrm{~J} / \mathrm{kgK}]$ & Heat capacity & $1.183^{*} 10^{3}$ & $1.039^{*} 10^{3}$ \\
$\lambda[\mathrm{W} / \mathrm{Km}]$ & Thermal conductivity & $6.9^{*} 10^{-2}$ & $2.59^{*} 10^{-2}$ \\
$\mu[\mathrm{Pas}]$ & Dynamic viscosity & $5.8^{*} 10^{-4}$ & $1.77^{*} 10^{-5}$ \\
$\alpha[1 / \mathrm{K}]$ & Thermal expansion coefficient & $1.8^{*} 10^{-3}$ & $3.35^{*} 10^{-3}$ \\
$D_{g}\left[\mathrm{~m}^{2} / \mathrm{s}\right]$ & Diffusion coefficient in the gas & & $6.98^{*} 10^{-6}$ \\
$\gamma_{T}[\mathrm{~N} / \mathrm{Km}]$ & T-derivative of surface tension & $-1.14^{*} 10^{-4}$ \\
$p_{s a t}[\mathrm{bar}]$ & Saturation pressure at $25^{\circ} \mathrm{C}$ & $2.69^{*} 10^{-1}$ \\
$\varepsilon_{g}[-]$ & Molar solutal expansion & 7.62 & \\
& coefficient in the gas & & \\
$L[\mathrm{~J} / \mathrm{kg}]$ & Latent heat of evaporation & & $1.116^{*} 10^{5}$ \\
$\kappa_{l}\left[\mathrm{~m}^{2} / \mathrm{s}\right]$ & Thermal diffusivity & $3.94 * 10^{-8}$ \\
$\gamma[\mathrm{N} / \mathrm{m}]$ & Surface tension & $13.6^{*} 10^{-3}$ \\
$M[\mathrm{~kg} / \mathrm{mol}]$ & Molar mass & 0.25 \\
$g\left[\mathrm{~m} / \mathrm{s}^{2}\right]$ & Gravity acceleration & 9.81 & \\
\hline
\end{tabular}




\section{Methods}

\subsection{Numerical considerations}

In this subsection, we discuss some properties and characteristics related to the numerical part of this work and some basic principles of the numeric method used. The numerical simulations have been performed by the software ComSol, which uses the finite element method (FEM). This method is based on discretizing the calculation domain into several elements. Subdividing the domain of interest into finite elements allows creating a generic element where the dependent variables are approximated at nodal points through interpolation functions. Connecting these elements to one another creates a mesh. In this work, we connected the elements in such a way that we have tetrahedral meshes, which appears to give the most stable configuration for fluid flow. The meshing at the liquid-gas interface is the densest one, where the most complicated fluid physics occurs. Information about these procedures can be found in, for instance (Li et al., 1999).

The solver is a fully coupled solver that solves the governing equations simultaneously as a set, or vector, of equations. A variable time stepping is used, depending on the convergence of the iterations at each time step. A relative convergence criterion of $10^{-4}$ is maintained.

Two types of meshing densities can be defined here. The first is the density at the surface, which is the number of elements at the liquid-gas interface per square $\mathrm{mm}$. The second is the number of elements in the bulk liquid and gas per cubic mm. These two densities can be chosen separately. It is important to verify whether the meshing density is sufficient for the simulations to be performed. For this purpose, we have changed the meshing density of the system and calculated at each meshing density the evaporation rate as a function of physical (not computing) time (the physical times are limited to 10 seconds). The changing of the meshing density has been done by increasing the density at the surface from 8 to 64 elements per $\mathrm{mm}^{2}$, and by increasing the density in the bulk (both gas and liquid phases) from 0.3 to 8 elements per $\mathrm{mm}^{3}$. This convergence study is also accompanied by a visualization of the patterns that are formed at the liquid-gas interface as a function of the meshing densities. The maximum computing time that we accept per simulation is 7 days, using an AMD Opteron Processor with $128 \mathrm{~GB}$ memory. The results of this convergence study is presented in Section 4.

\subsection{Experimental setup}

The experimental setup that is used is presented in detail in (Lyulin and Kabov, 2014). In this subsection, we will mention succinctly some important features. The test cell consists of two superimposed Plexiglas units which are appropriately shaped to create the gas channel and the liquid cell. The test cell is shown in Fig. 2.

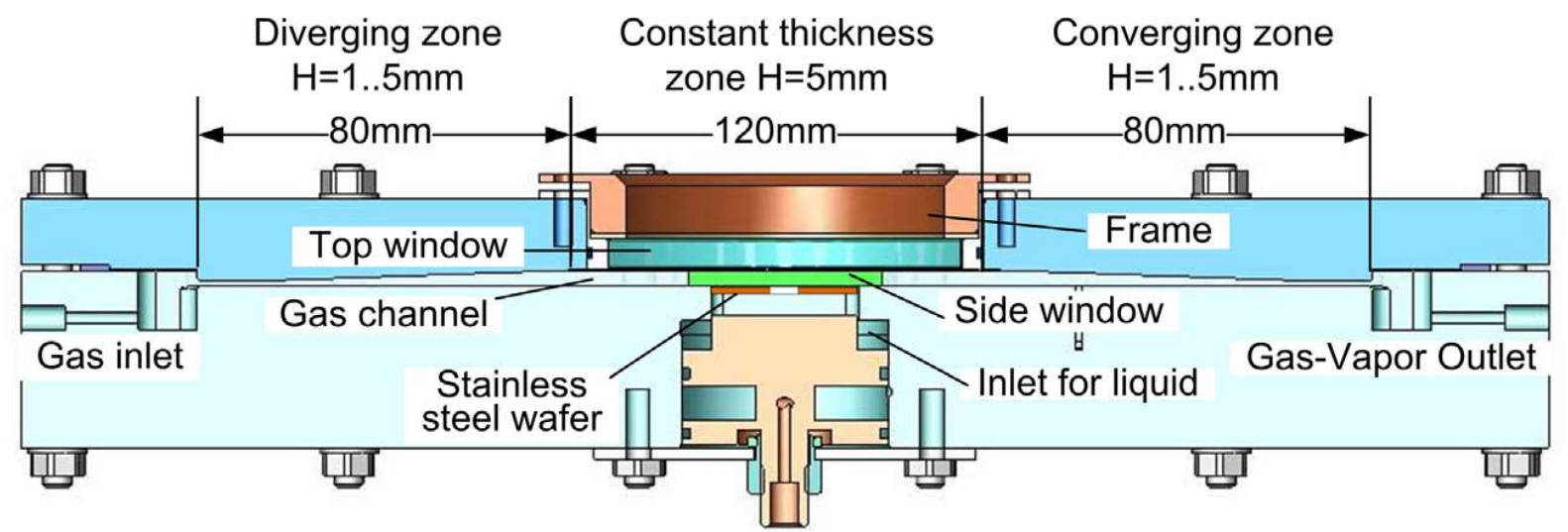

Figure 2. Longitudinal cross section of the Test Cell. Reprinted from (Lyulin and Kabov, 2014) with permission from Elsevier. 
The test cell is a flat rectangular channel of $440 \times 100 \times 5 \mathrm{~mm}^{3}$ (length $\mathrm{x}$ width $\mathrm{x}$ depth) and is cut out in an organic glass block. There are two distribution chambers, each with a longitudinal section of $20 \times 20 \mathrm{~mm}^{2}$, cut out at the inlet and at the outlet of the channel. A rectangular reservoir of section $50 \times 50 \mathrm{~mm}^{2}$ is cut in the middle part of the channel. Its bottom is formed by a polished copper piston surface. The depth of the reservoir can be chosen in the range of 1.5 to $8 \mathrm{~mm}$ due to a vertically adaptable piston. The channel has a transparent upper cover of organic glass with two wedge-shaped lugs on its lower side. There are three zones in the gas channel: the inlet diverging zone, where height of the channel increases from $1 \mathrm{~mm}$ to $5 \mathrm{~mm}$ within a distance of $80 \mathrm{~mm}$; the middle constant-thickness zone of height $5 \mathrm{~mm}$ and of $120 \mathrm{~mm}$ length; the outlet converging zone, where height of the channel reduces from $5 \mathrm{~mm}$ to $1 \mathrm{~mm}$ within a distance of $80 \mathrm{~mm}$. Due to this geometry of the channel, a uniform distribution of the gas flow in the middle zone is achieved. Two glass insets are glued into the flank walls of the channel enabling optical observations of the gas and vapor flow. A frame is provided in the cover to maintain a transparent window of $120 \mathrm{~mm}$ diameter. In order to adapt the setup to the original liquid chamber of the test cell that is used in the EP experiment a special inset unit (Fig. 3 ) is used.

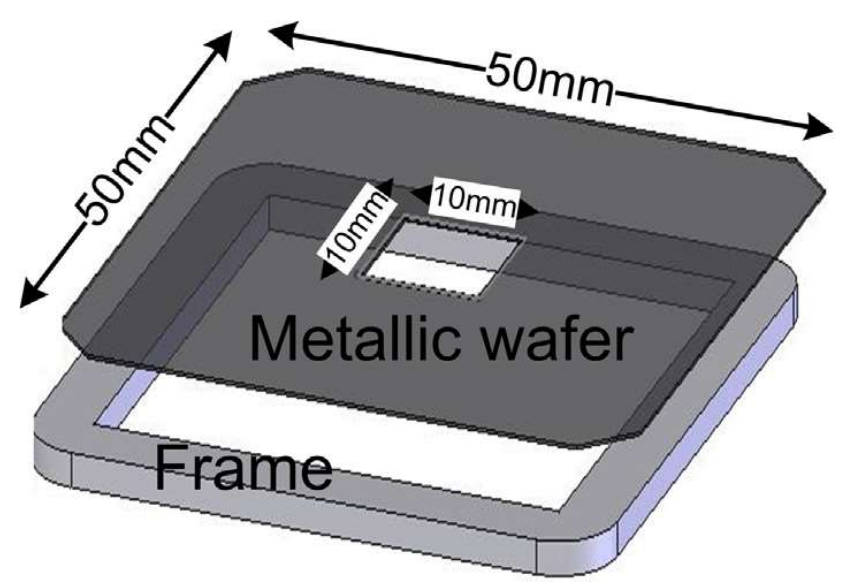

Figure 3. Design of the inset unit for the Test Cell. Reprinted from (Lyulin and Kabov, 2014) with permission from Elsevier.

The inset is sealed to the liquid chamber. The unit consists of a stainless steel frame and a metallic wafer with a micro-groove around the opening. The micro-groove is used in order to prevent spreading of the working liquid over the metallic wafer. Preventing liquid from spilling out over the wafer surface is achieved due to the sharp edge (edge effect) bordering the liquid in the opening. Using a sharp edge as barrier for liquid spreading is proposed by Gibbs (1961) and further elaborated from a thermodynamic perspective in the work of Fang and Amirfazli (2012). In the work of Grishaev et al. (2013), the ability of the micro-groove to prevent the spreading of working fluid (HFE-7100) having low surface tension on a surface is studied. The authors show that the micro-groove can be used to prevent spreading of the working liquid. A contact angle of up to $50^{\circ}$ has been measured. The geometry of the liquid chamber, on which the numerical model is base, is shown in Fig. 4. 


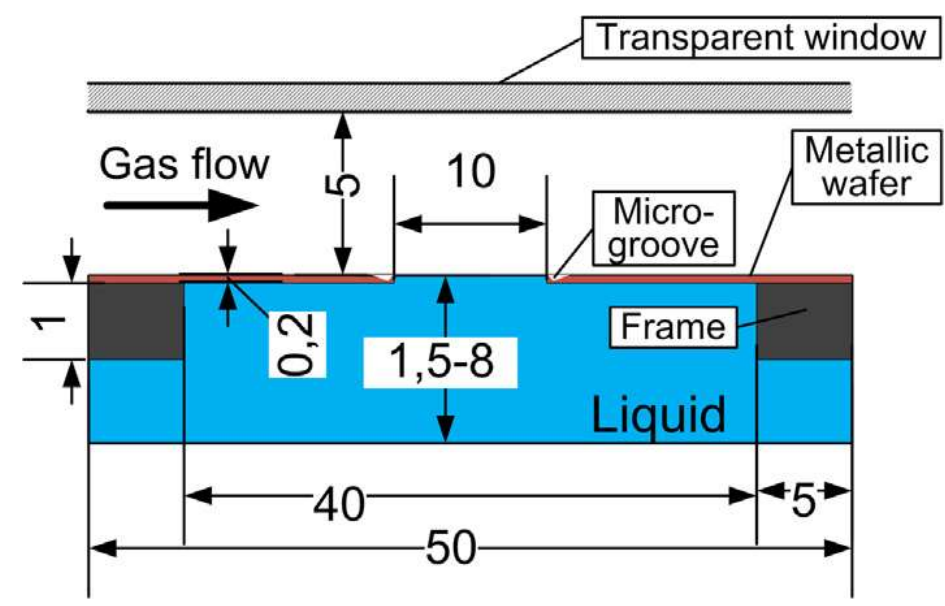

Figure 4. Geometry of the liquid chamber (all sizes are in $\mathrm{mm}$ ). Reprinted from (Lyulin and Kabov, 2014) with permission from Elsevier.

The gas flow coming into the channel through the fitting at the inlet and through the gasdistributing chamber runs over the free surface of the evaporating liquid that is filled into the reservoir. The gas and vapor mixture leaves the channel through the outlet distributing chamber and through the fitting at the outlet. The required temperature of the working liquid inside the reservoir is maintained with the help of the piston. The temperature of the piston is controlled by water that is supplied from a thermostatic bath through the inner channels of the piston.

\section{Results}

Subsection 4.1 presents a convergence study to assess the meshing quality and the convergence of the numerical results. In subsection 4.2, we proceed with the discussion of the evaporation rate and the influence of several parameters. We will also validate these results by the experiments. Subsection 4.3 explains the found trends by the simulations.

\subsection{Convergence study}

The convergence study presented in this subsection is performed for a liquid thickness of $5 \mathrm{~mm}$, a temperature of $20^{\circ} \mathrm{C}$ and a gas flow of $500 \mathrm{ml} / \mathrm{min}$. It should be noted that, even though not presented here, the same conclusions can be drawn for the other values of the parameters. Fig. 5 presents the average evaporation rate over the whole liquid-gas interface as a function of time for different surface meshing densities with a bulk meshing density of 8 elements per $\mathrm{mm}^{3}$. Fig. 6 presents the same, but for different bulk meshing densities with a surface meshing density of 64 elements per $\mathrm{mm}^{2}$. 


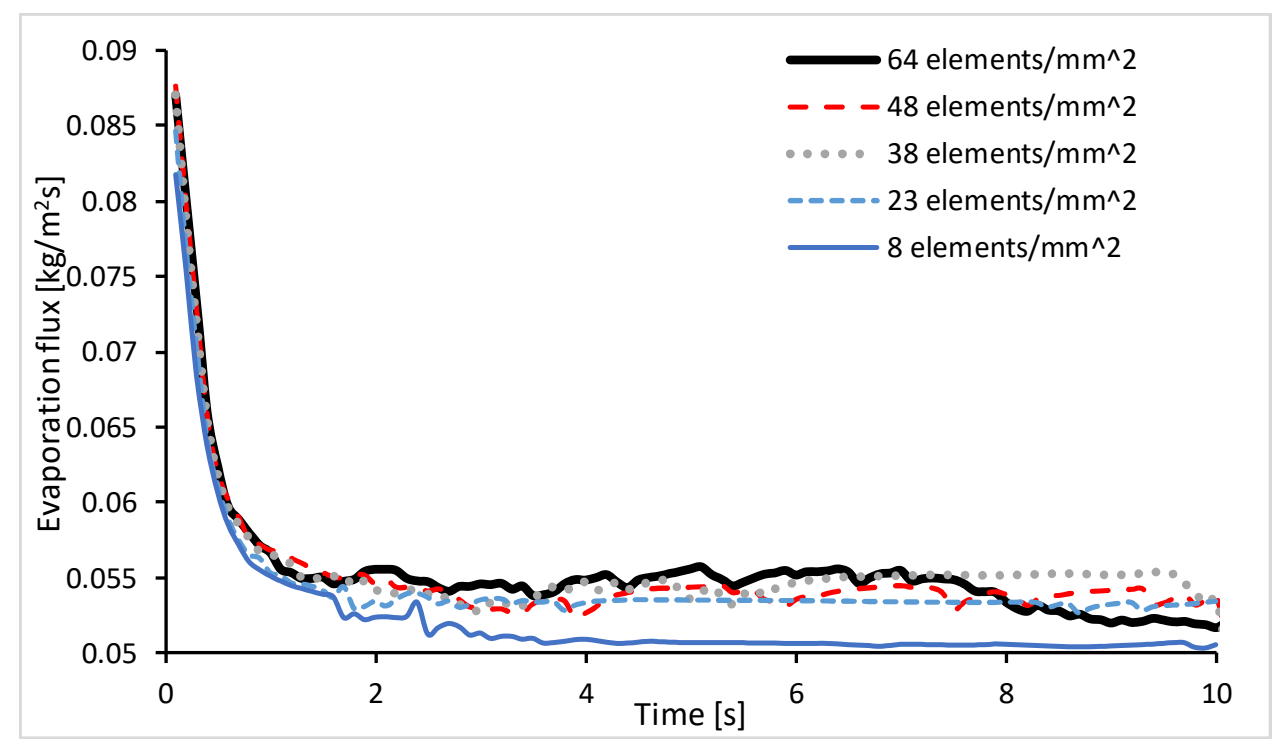

Figure 5. The evaporation rate corresponding to different meshing densities at the surface with bulk meshing density of 8 elements per $\mathrm{mm}^{3}$.

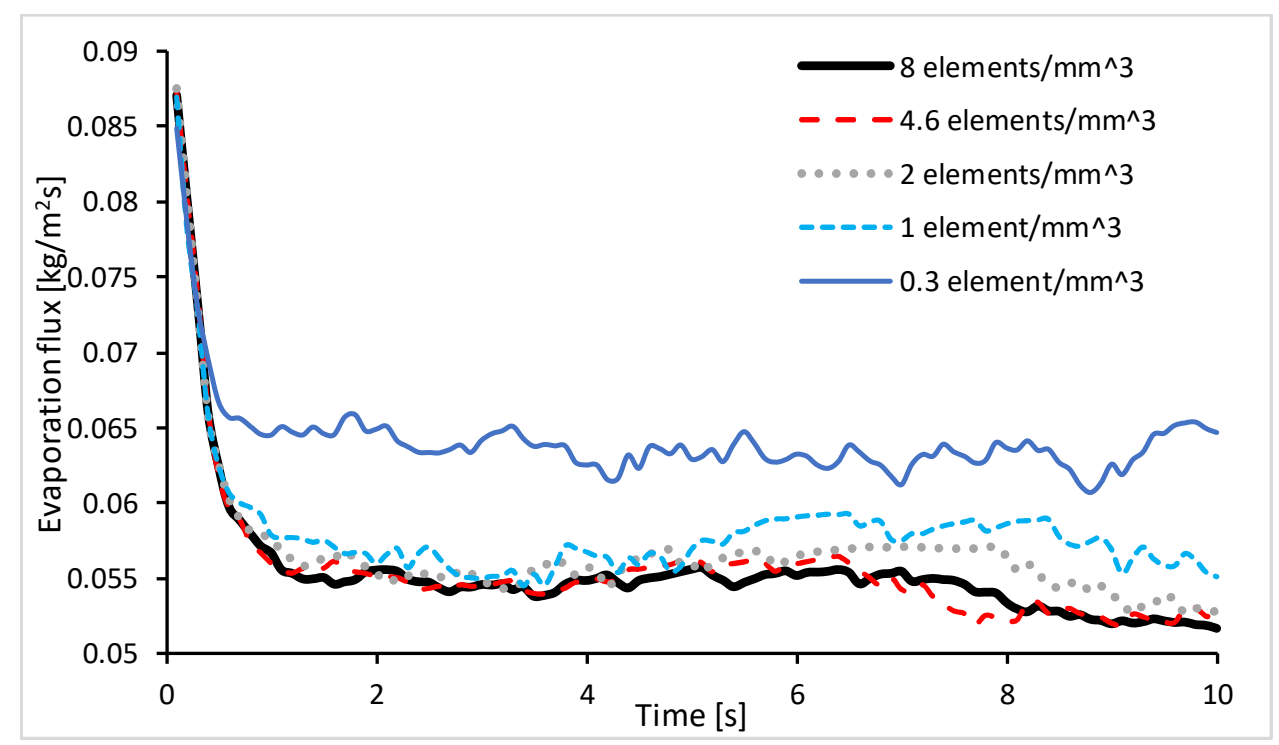

Figure 6. The evaporation rate corresponding to different meshing densities in the bulk with surface meshing density of 64 elements per $\mathrm{mm}^{2}$.

We can note from Fig. 5 that for the evaporation flux an acceptable convergence is obtained. This convergence is only qualitative, since no quantitative convergence can be obtained in the context of the present work. Indeed, the thermal convection patterns are highly non-linear and therefore an even very small change in the initial meshing density can give different values (as is the case in chaos theory applicable to fluid dynamics). Nevertheless, we can observe that from 23 to 64 elements per $m m 2$ the evaporation flux oscillates around approximately the same value so that we can consider a surface meshing density of 64 elements $/ \mathrm{mm}^{2}$ satisfactory. Fig. 6 , however, shows that only bulk meshing densities of 4.6 and 8 elements $/ \mathrm{mm}^{3}$ seem to give evaporation fluxes that oscillate around approximately the same value. For this reason, we find 8 elements $/ \mathrm{mm}^{3}$ a reasonable bulk meshing densities. Next to this, to give an idea of how these meshing densities influence the patterns on the liquid-gas interface, we also present snapshots of temperature patterns at the liquid-gas interface at $t=0.1,1$ and $8 \mathrm{~s}$. These times are chosen because, on observing all the results, it came to our attention that the patterns at the aforementioned respective times are clearly different. Therefore, performing a qualitative 
convergence study, it is only beneficial to do this for different pattern forms (note that it is, however, not the purpose to analyse the pattern forms as such). The results are presented in Figs. 7 and 8 (obtained for the same set of parameters as for Figs. 5 and 6).

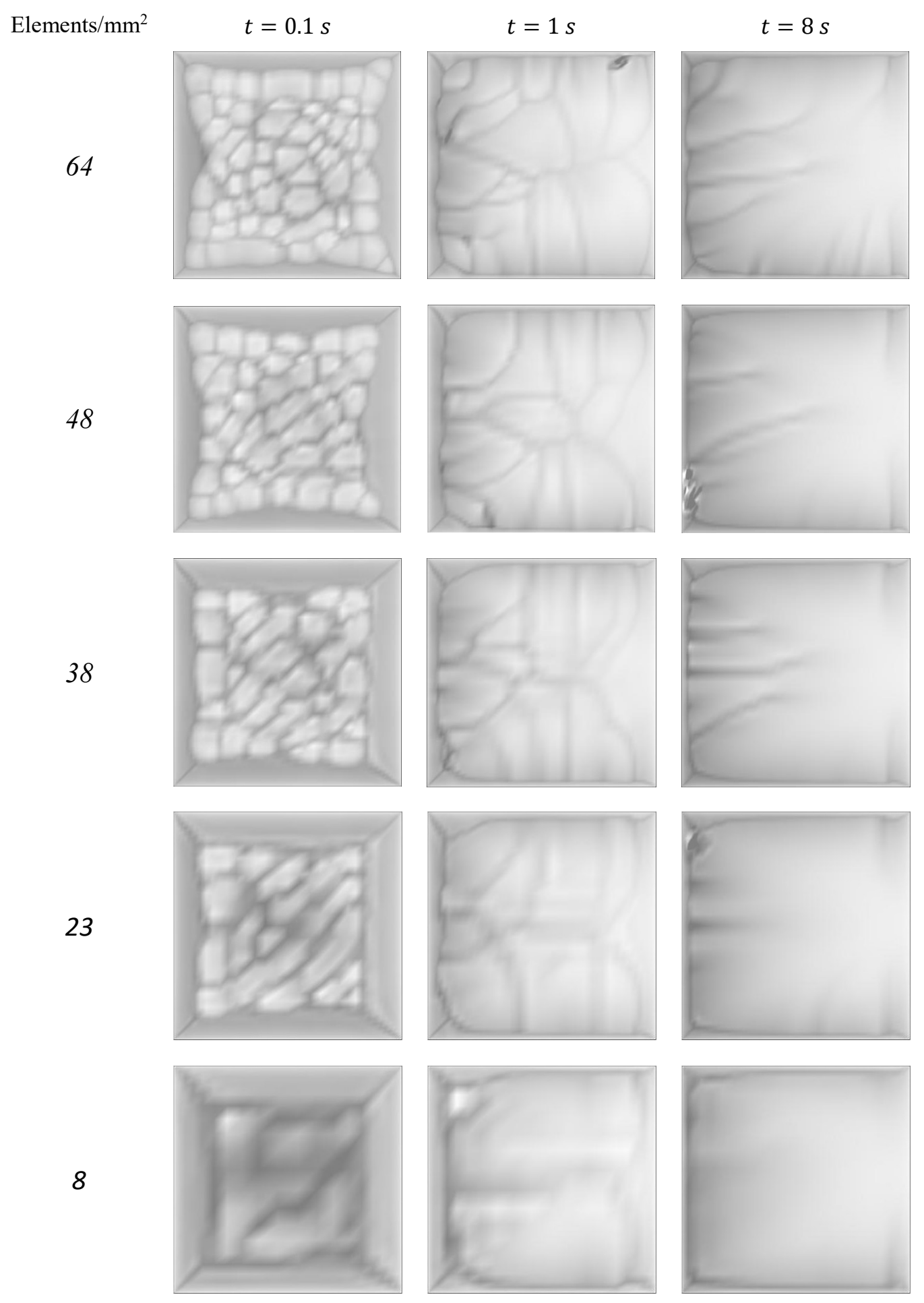

Figure 7. The temperature patterns for different meshing densities at the surface with bulk meshing density of 8 elements per $\mathrm{mm}^{3}$. Temperature ranges from 298.15 (light colored) to 291 (dark colored) $\mathrm{K}$.
Elements $/ \mathrm{mm}^{3}$
$t=0.1 \mathrm{~s}$
$t=1 s$
$t=8 \mathrm{~s}$ 

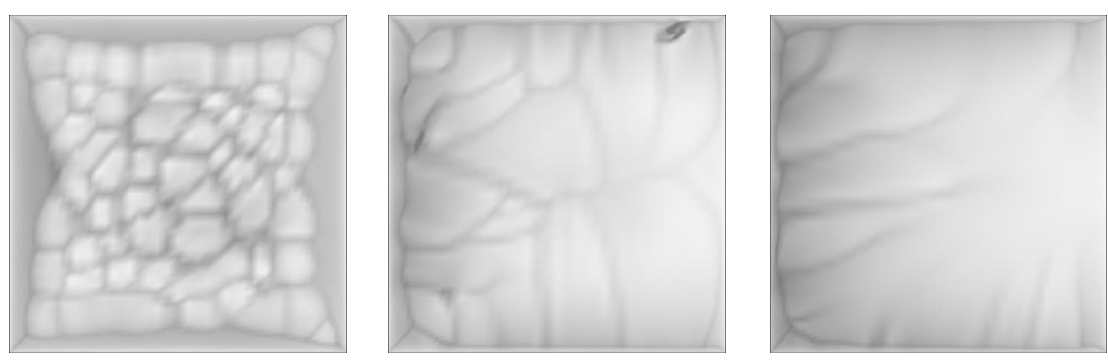

4.6
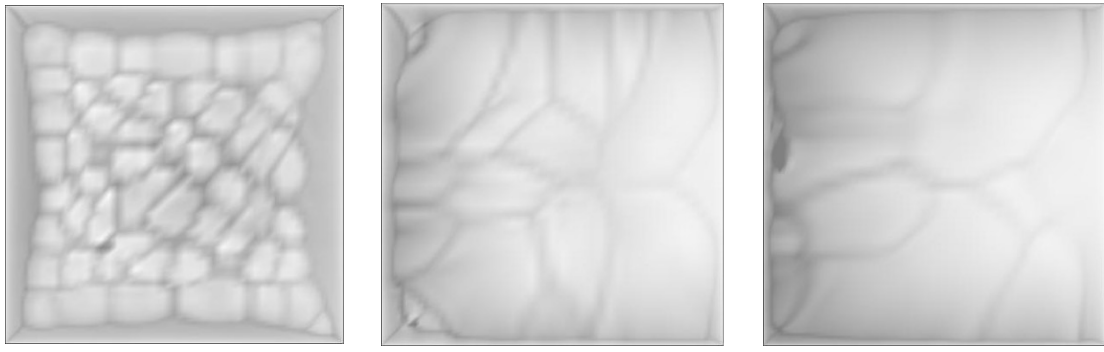

2
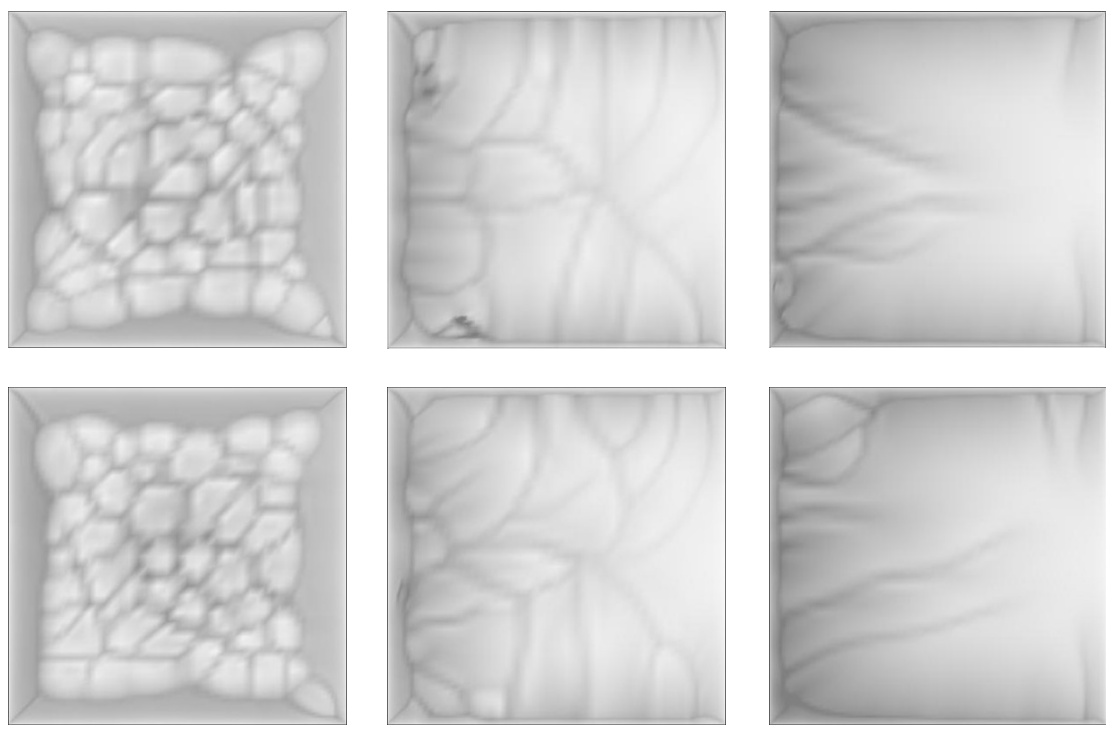

1
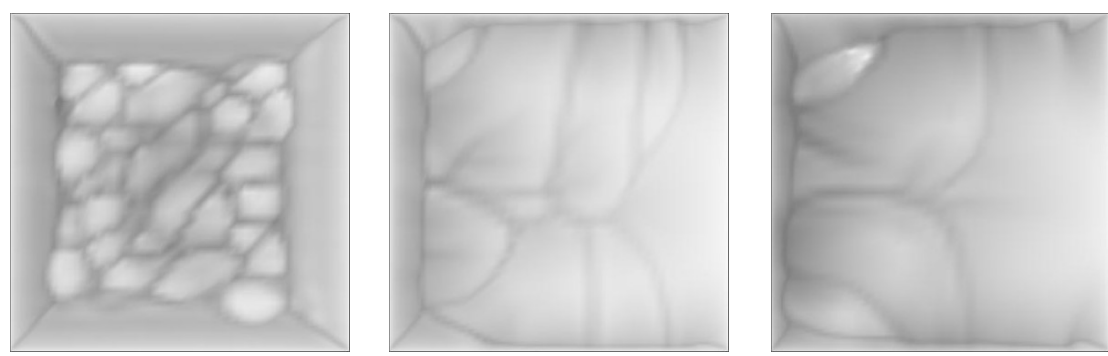

Figure 8. The temperature patterns for different meshing densities in the bulk with surface meshing density of 64 elements per $\mathrm{mm}^{2}$. Temperature ranges from 298.15 (light colored) to 291 (dark colored) K.

It appears that the patterns with surface meshing densities of 64 and 48 elements $/ \mathrm{mm}^{2}$, at each respective time, correspond well with each other, suggesting good qualitative convergence. The same conclusion can be drawn when comparing the bulk meshing densities of 8 and 4.6 elements $/ \mathrm{mm}^{3}$. We can see that the convergence of the evaporation rate depends more on the meshing density of the bulk phase than on that of the surface, which can be explained by the fact that the evaporation rate is linked by the concentration gradients in the bulk phases (and not by the horizontal gradients on the liquid-gas interface, which are rather a consequence of 
the evaporation). We can also conclude from this that the temperature gradients (forming the patterns) at the surface depend obviously on the surface meshing density. We can finally state that increasing the surface and bulk meshing densities from 48 to 64 pixels per $\mathrm{mm}^{2}$ and from 4.6 to 8 packs per $\mathrm{mm}^{3}$, respectively, hardly changes significantly the temperature pattern structure and evaporation flux. Note that the temperature patterns should be taken qualitatively since this highly non-linear process is highly sensible to numeric perturbations. For final consideration on the convergence study, Fig. 9 shows the computing time as a function of the meshing densities.

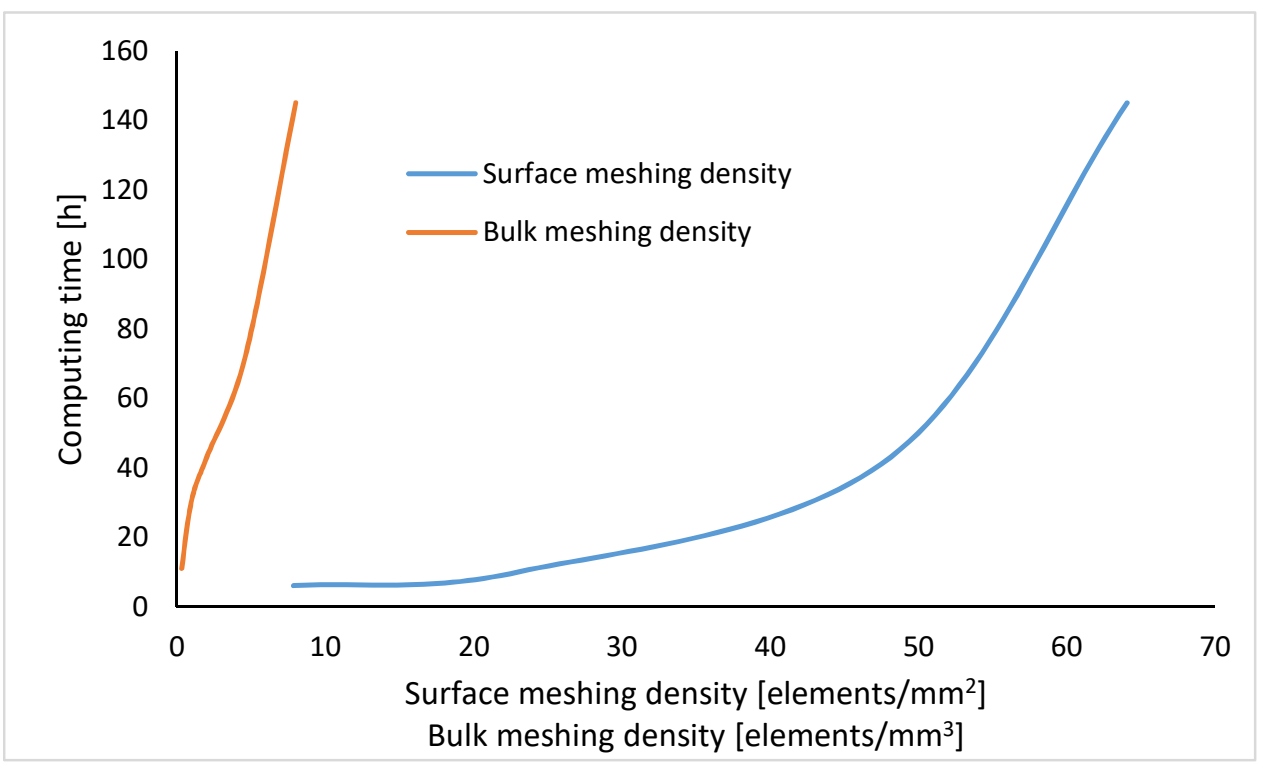

Figure 9. Computing time as a function of the surface meshing density (with bulk meshing density of 8 elements $/ \mathrm{mm}^{3}$ ) and bulk meshing density (with surface meshing density of 64 elements $/ \mathrm{mm}^{2}$ ).

We can see that at surface and bulk meshing densities of 64 elements per $\mathrm{mm}^{2}$ and 8 elements per $\mathrm{mm}^{3}$, respectively, the computing time (for a physical time of 10 seconds) increases steeply to 145 hours (which we find already time consuming for a parametric study), and the convergence seems to be satisfactory. Therefore, we will perform the rest of our simulations in this paper with the last-mentioned meshing densities.

\subsection{Evaporation rate}

Figures 10 to 12 show the average evaporation rate through the liquid-gas interface as a function of the gas flow, temperature and liquid thickness from the numerical simulation, indicated by lines. In the same figures, the corresponding values from the experiments are shown, denoted by markers. The evaporation rates (besides averaged over the liquid-gas interface) are also averaged over the last $4 \mathrm{~s}$ of the simulation (which simulates an evaporation process of $10 \mathrm{~s}$ ). We have noted that over the last $4 \mathrm{~s}$ the values of the average evaporation rate, although fluctuating around $5 \%$ of the mean value, does not change significantly. Therefore, we assume that in that period the system has come to a quasi-stationary state, corresponding to that of the experiments. Fig. 10 shows the evaporation rate as a function of the temperature imposed at the boundaries of the system while Fig. 11 shows its dependence on the gas flow and Fig. 12 on that of the liquid thickness. 


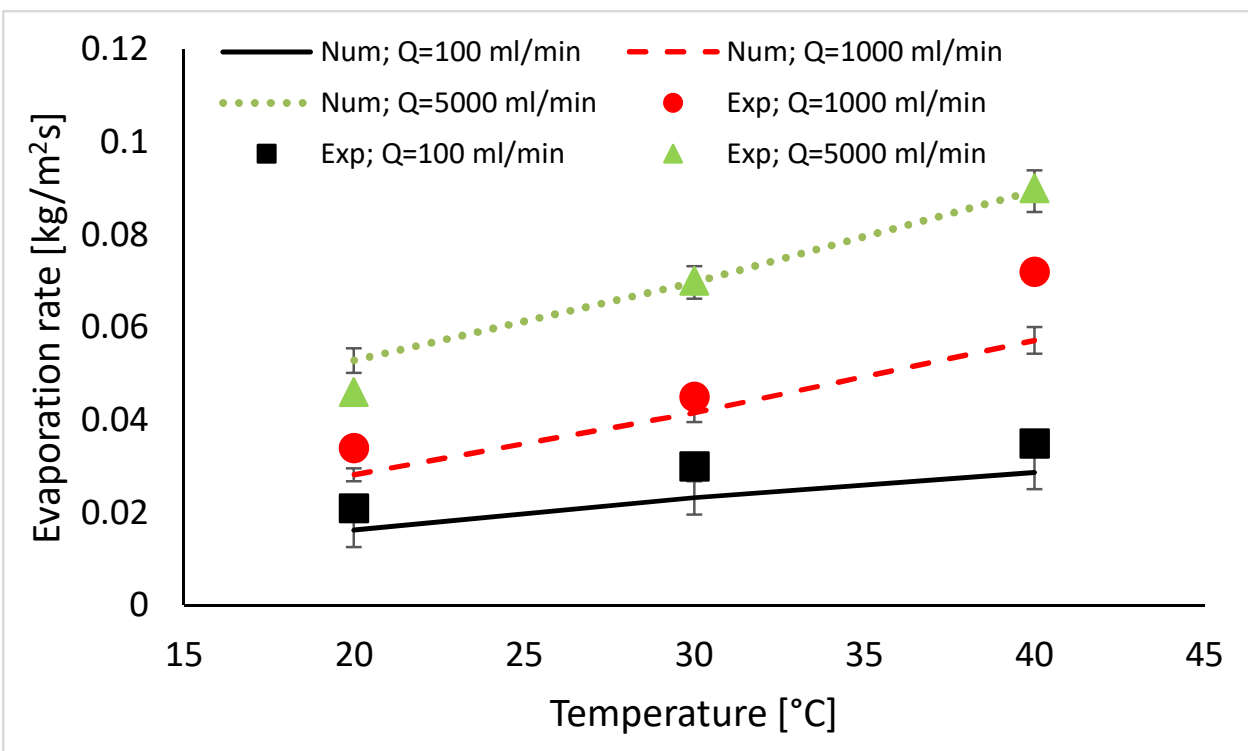

Figure 10: Evaporation rate as a function of the temperature for several gas flow rates and a liquid thickness of $3 \mathrm{~mm}$. The error bars on the curves correspond to the $5 \%$ discussed in the text.

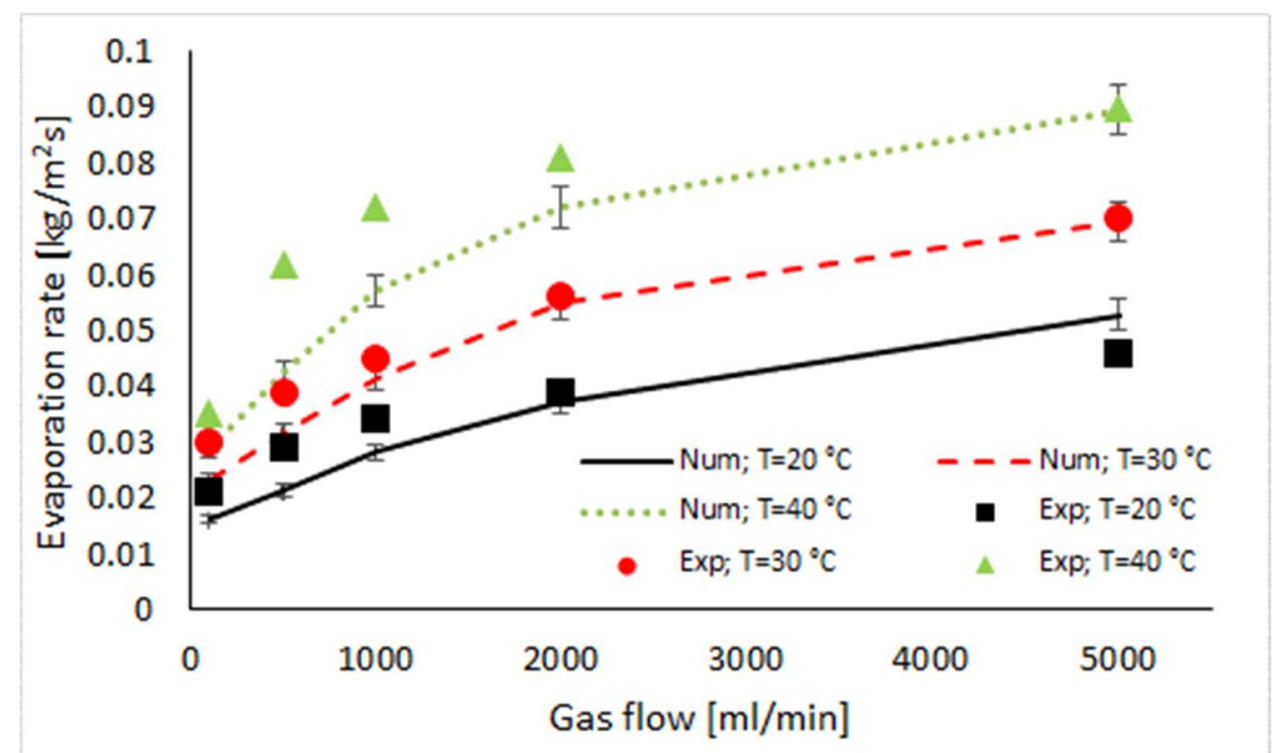

Figure 11: Evaporation rate as a function of the gas flow for several temperatures and a liquid thickness of $3 \mathrm{~mm}$. The error bars on the curves correspond to the $5 \%$ discussed in the text. 


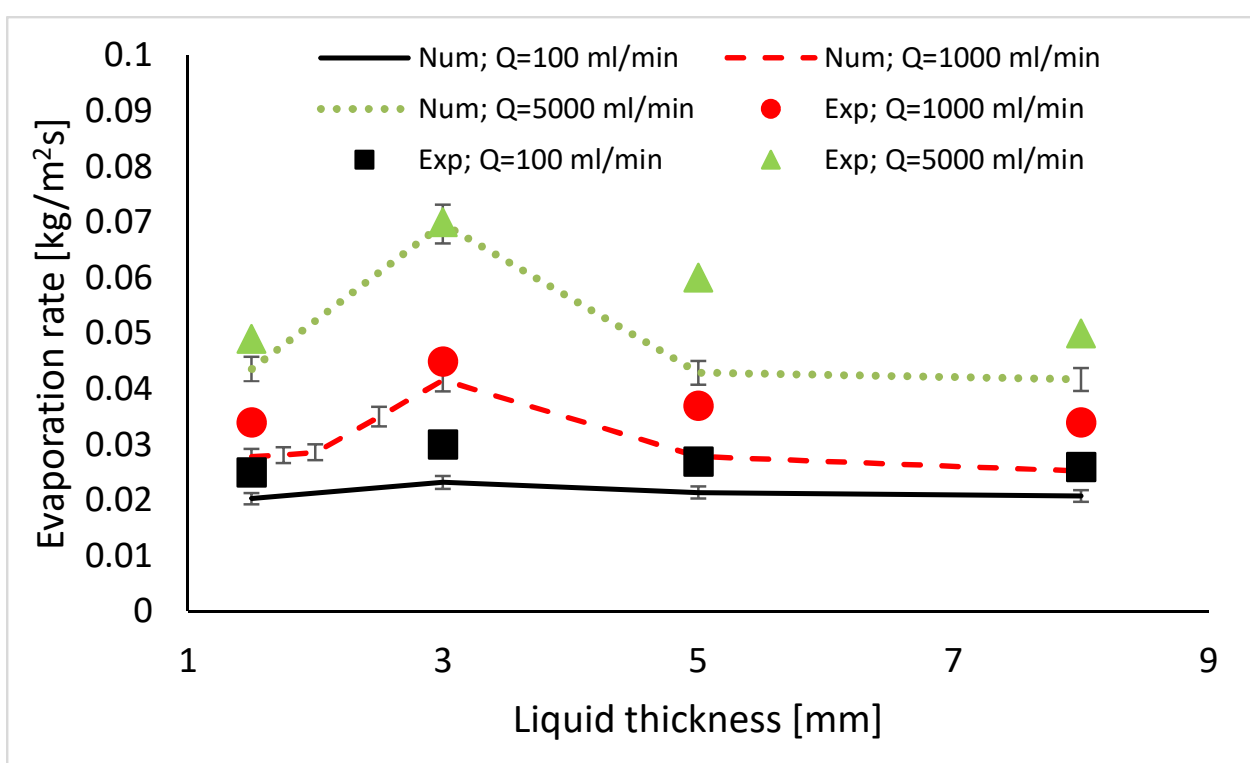

Figure 12: Evaporation rate as a function of the liquid thickness for several gas flows and a temperature of $30{ }^{\circ} \mathrm{C}$. The error bars on the curves correspond to the $5 \%$ discussed in the text.

First of all, the simulations correspond rather well to the experimental values, which, together with the convergence study, secures a certain accuracy of the simulations. It can be seen that the evaporation rate increases monotonically as a function of the temperature and the gas flow. An increase in the temperature (Fig. 10) will not only increase the molar fraction of the HFE7100 vapor (due to a higher saturation pressure) at the liquid-gas interface (see Eq. (20)), but also increases the convection. The latter increases the kinetic energy of the liquid molecules that are able to leave easier the liquid-gas interface. The increase of the evaporation rate with the gas flow rate (Fig. 11) is linked with a diminishing gas boundary layer at the liquid-gas interface. This increases the concentration gradient at the interface and thus the evaporation rate (see Eq. (16)). The dependence on the gas flow rate is non-linear, but in a decreasing way, i.e. it would be correlated to the gas flow rate with an exponent smaller than one. The effect on the liquid thickness (Fig. 12) is, however, rather different. We can observe a certain maximum around a liquid thickness of $3 \mathrm{~mm}$. Note that in Fig. 12, more points are calculated for a gas flow of $1000 \mathrm{ml} / \mathrm{min}$ and an initial temperature of $30{ }^{\circ} \mathrm{C}$ for the simple reason that at these conditions the influence on the liquid thickness will be studied in more detail as a case study. Since the dependence of the evaporation rate on the liquid thickness is somewhat non-standard, we dedicate to it a separate subsection, where we discuss further the dependence of the evaporation rate on the liquid thickness.

\subsection{Analysis of the influence of the liquid thickness}

In this subsection, we will focus more on the effect of the liquid thickness, which has shown an interesting effect on the evaporation rate. Indeed, Fig. 12 shows that there is a local maximum around a liquid thickness of $3 \mathrm{~mm}$. More precisely we will analyse the results, and in particular, the structure of the flow, in terms of the thickness of the liquid layer. To prepare the discussion presented below, it is also important to introduce the "aspect ratio" of the fluid layer, which we define as the ratio between the dimension of the square opening $(10.6 \mathrm{~mm})$ and the thickness of the layer. Before the discussion below, it is also important to recall that evaporation induces a cooling of the surface, which induces a temperature gradient from the top to the bottom of the layer. Consequently, Rayleigh-Bénard-Maragoni instabilities and the associated cellular convection will be present in the system. Moreover, due to the gas flow imposed in the upper gas, horizontal gradients will also be generated in the fluid, which will in turn induce large- 
scale convection. The global behaviour of the system that will be discussed below will thus be influenced by all these mechanisms.

To start the discussion, let us describe in some detail the convective structure in the following particular situation. Fig. 13 corresponds to a thickness equal to $3 \mathrm{~mm}$ (which corresponds to an aspect ratio equal to 3.53 ), a gas flow of $1000 \mathrm{ml} / \mathrm{min}$, and a temperature of $30{ }^{\circ} \mathrm{C}$. In the figure, the surface velocity parallel to the gas flow is represented in several snapshots, from the beginning of the calculation to $\mathrm{t}=10 \mathrm{~s}$. The yellow-red areas correspond to fluid moving in the direction of the gas flow, while green-blue zones must be associated with a "counter flow" with respect to the upper gas. Note that we have checked that the values of the surface velocity parallel to the gas are in average much higher than the surface velocity perpendicular to the gas flow. Therefore, we can only consider the horizontal surface velocity parallel to the gas flow in the discussion. One can also observe that there is a transient phase in Fig. 13, where the pattern of the liquid-gas interface changes considerably. However, from $t=6$ to $10 \mathrm{~s}$, the structure of the flow remains more or less stationary, with a clearly stable red "vertical" strip on the left, and also another red-yellow "vertical" strip in the middle of the surface. Of course, this general pattern is perturbed by bursts of chaotic behavior taking the form of red spots that seem to detach from the right strip, breaking it for some time, and move on the surface. 


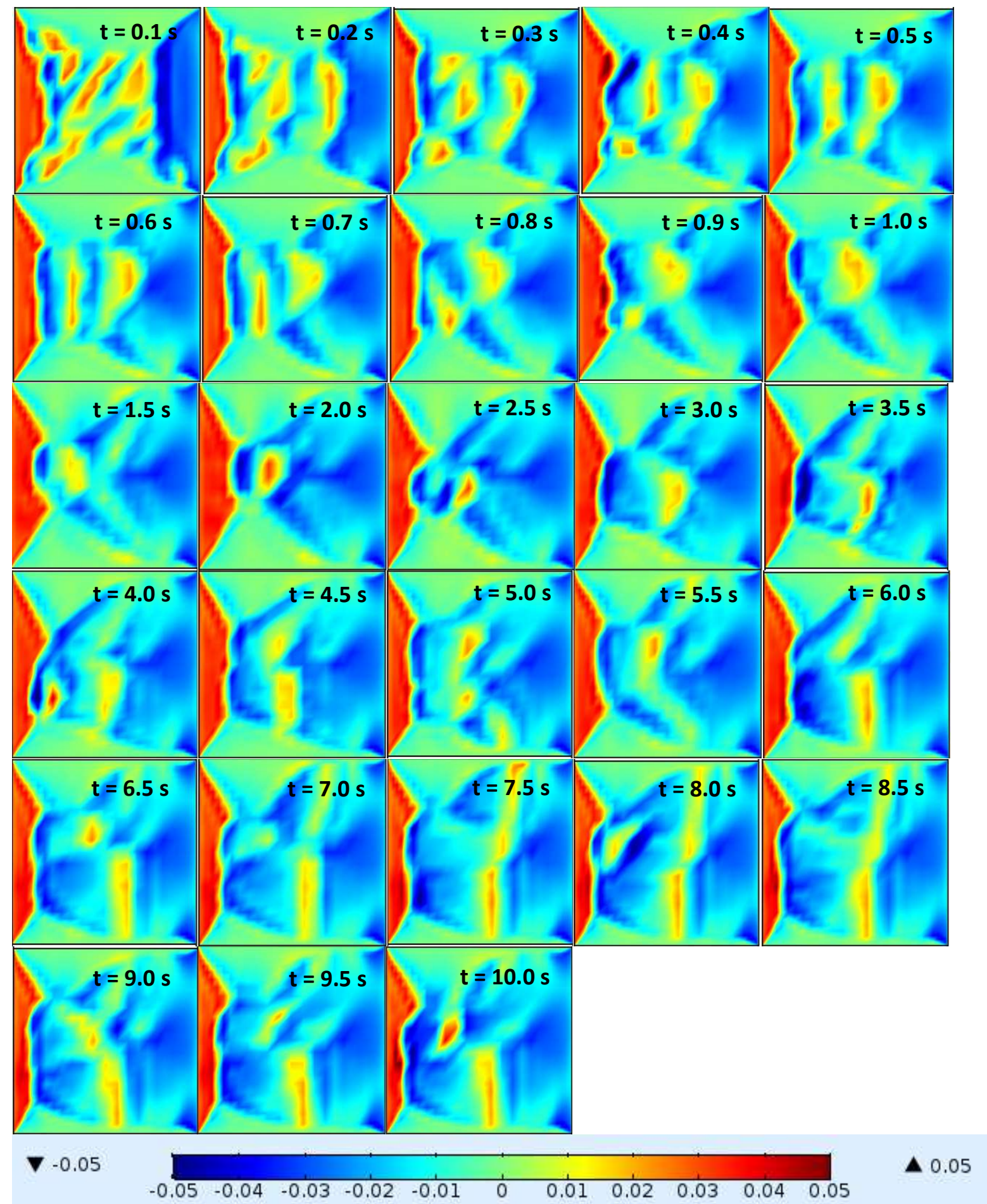

Figure 13. Top view of the horizontal velocity profile parallel to the gas flow (velocity in $\mathrm{m} / \mathrm{s}$ ) at the liquid-gas interface for a gas flow rate of $1000 \mathrm{ml} / \mathrm{min}$, an initial temperature of $30{ }^{\circ} \mathrm{C}$ and a liquid thickness of $3 \mathrm{~mm}$ for different time snapshots.

Let us now consider different thicknesses, corresponding to different aspect ratios, with the same gas flow and temperature. Fig. 14 shows the influence of the liquid thickness on the horizontal velocity at the liquid-gas interface, parallel to the gas flow direction, for a gas flow rate of $1000 \mathrm{ml} / \mathrm{min}$ and a temperature of $30^{\circ} \mathrm{C}$. The times corresponding to the different images are always larger than $6 \mathrm{~s}$ in order to make sure that a "stationary" state has been reached and 
the precise instant of each picture is chosen in order that the corresponding picture be representative of this "stationary" behaviour, that will be discussed in more detail now.

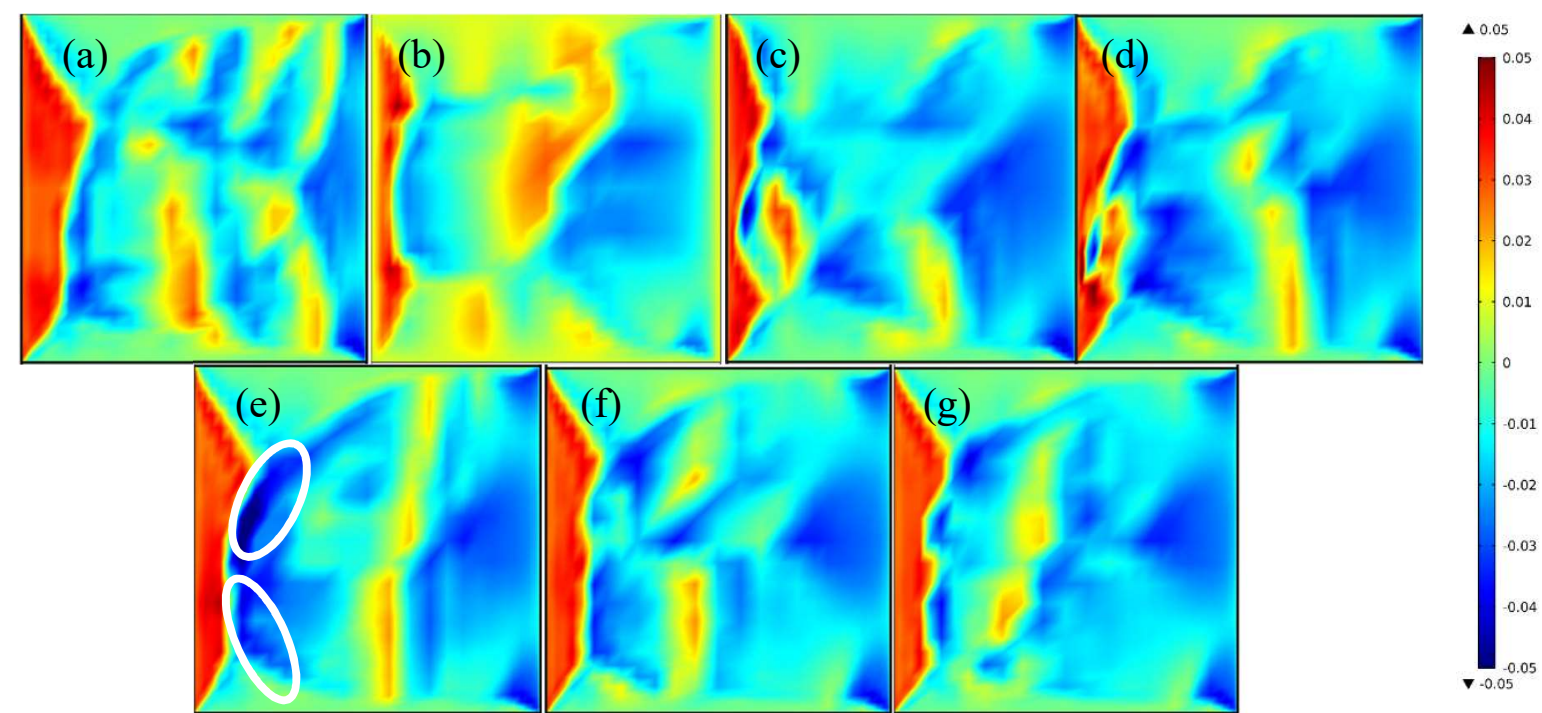

Figure 14. Top view of the horizontal velocity profile parallel to the gas flow (velocity in $\mathrm{m} / \mathrm{s}$ ) at the liquid-gas interface for a gas flow rate of $1000 \mathrm{ml} / \mathrm{min}$, an initial temperature of $30{ }^{\circ} \mathrm{C}$ and a liquid thickness of (a) $1.5 \mathrm{~mm}$, (b) $1.75 \mathrm{~mm}$, (c) $2 \mathrm{~mm}$, (d) $2.5 \mathrm{~mm}$, (e) $3 \mathrm{~mm}$, (f) $5 \mathrm{~mm}$ and $(\mathrm{g}) 8 \mathrm{~mm}$.

Fig. 14 shows that for all aspect ratios, kind of vertical strips, more or less regular, sometimes broken, and from which several spots sometimes detach, can be observed, as in the last snapshots of Fig. 13. Moreover, and this is an important result, the number of these vertical strips changes with the aspect ratio of the fluid layer, and more precisely decreases when the aspect ratio decreases (i.e. the liquid thickness increases from 1.5 to $8 \mathrm{~mm}$ ). This result is also nicely illustrated in Fig. 15 which provides vertical cross sections of the flow and shows the change in the number of convective "rolls" making up the pattern. 


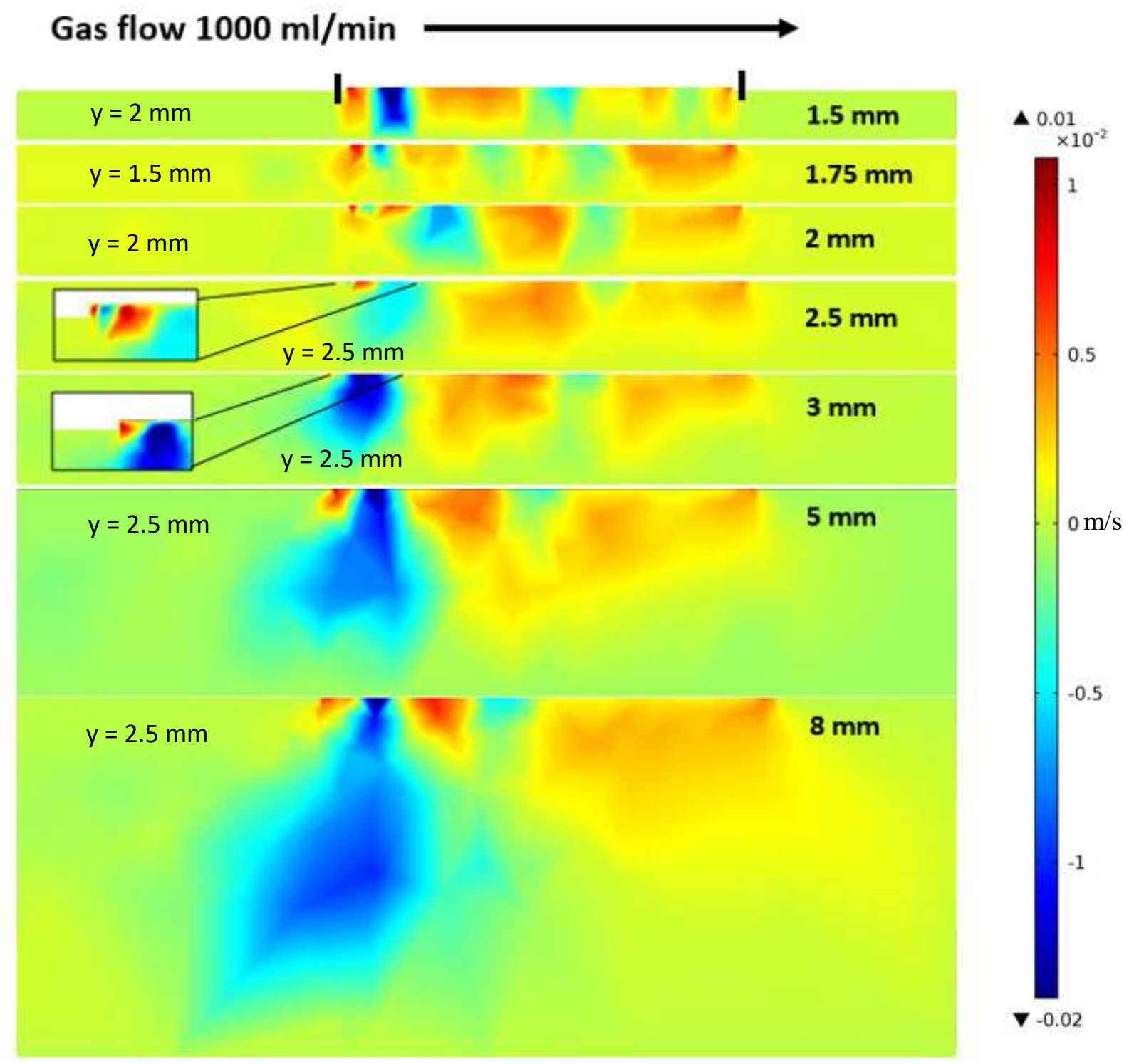

Figure 15. Liquid vertical velocity for several liquid thicknesses for a gas flow rate of 1000 $\mathrm{ml} / \mathrm{min}$ and a temperature of $30^{\circ} \mathrm{C}$. The small vertical lines crossing the upper surface represent the borders of the liquid-gas interface opening. The direction and value of the gas flow are indicated by an arrow above the figure, the corresponding liquid thicknesses and the $y$ coordinates (perpendicular to the gas flow, with $y=0$ corresponding to left bottom corner of the images in Fig. 14) of the slices are indicated on the images.

Fig. 15 shows that at a liquid thickness of $1.5 \mathrm{~mm}$, there are the maximum number of cells caused by liquid fingers due to an evaporation-cooled liquid-gas interface (represented by lower temperature areas in the image). It is interesting to notice that this maximum number of cells also corresponds to the maximum number of strips shown in Fig. 14. At a liquid thickness of 3 $\mathrm{mm}$ and higher, a lower number of cells is apparent. So, it appears that as the liquid thickness increases (the aspect ratio decreases), the number of cells decreases, with a clear change occurring for a liquid thickness around 2.5 or $3 \mathrm{~mm}$. We will now explain that this result is a consequence of confinement effects due to the cover sheet. Confinement effects have already been studied in details (Dauby and Lebon, 1996; Dauby et al., 1997; Dauby et al., 2000) in the case of vertical no-slip boundaries. An important result of these analyses was the dependence of the number of convective cells with respect to the aspect ratios of the container. It is also shown that the number of cells is in fact such that the observed cells are neither too much 
compressed, nor too dilated and have more or less the 'natural' dimension they would have in an infinite layer. Another result of these papers was the discontinuity of the slope of the function linking the critical Marangoni or Rayleigh numbers with the aspect ratios. This discontinuity is associated with local maxima of the critical curves and with changes in the number of cells, these cells being more and more dilated, or compressed, when the maximum is approached from one side or from the other. The problem studied here is of course not completely similar because the confinement is only imposed by the cover sheet at the liquid-gas interface, and not by a complete vertical wall. Moreover, we have also a horizontal gas flow that generates horizontal gradients, but the change in the number of cells observed in Figs. 14 and 15 is clearly reminiscent of the confinement effects recalled above. The discontinuity of the slope of characteristic quantities and the appearance of a local maximum is also observed in our problem, as illustrated in Fig. 12 above, which exhibits a maximum of the evaporation flux with respect to the liquid thickness or aspect ratio.

Now we will show that the gas flow also participates in the pattern selection mechanism. To this end, we have calculated the mean value (over the whole surface) $v_{a v}$ of the horizontal liquid velocity parallel to the gas flow. This quantity was found always negative, which corresponds to a global liquid counter flow at the liquid-gas interface (evaporation, and thus cooling, is more important on the left (entrance of the gas), because the vapor concentration is lower, which induces Marangoni convection). Fig. 16 shows the absolute value of $v_{a v}$ as a function of the liquid thickness for a gas flow of $1000 \mathrm{ml} / \mathrm{min}$ and an initial temperature of 30 ${ }^{\circ} \mathrm{C}$. A maximum is observed in the curve for thicknesses close to those defining the maximum in Fig. 12 and the change of the number of cells in Figs. 14 and 15. This coincidence reveals that the general structure of the flow, and in particular the number of cells, is also influenced by the gas flow taking place above the liquid.



Figure 16. Absolute value of the average horizontal velocity $\left(\left|v_{a v}\right|\right)$ parallel to the gas flow for different liquid thicknesses $\left(d_{l}\right)$ for a gas flow of $1000 \mathrm{ml} / \mathrm{min}$ and an initial temperature of 30 ${ }^{\circ} \mathrm{C}$

\section{Conclusions}

In this work, we studied the evaporation rate of an HFE-7100 liquid that evaporates into a nitrogen gas flow by means of three-dimensional simulations. The calculated evaporation rates are validated against experimental values from an experimental setup with the same dimensions 
and parameters. The three-dimensional numerical model is then used to explain some tendencies, especially related to the effect of the liquid thickness. The other parameters are the overall initial temperature and the gas flow rate. No humidity is considered in the gas flow. The temperatures are varied from 20 to $40{ }^{\circ} \mathrm{C}$, the gas flow rate from 100 to $5000 \mathrm{ml} / \mathrm{min}$ and the liquid thickness from 1.5 to $8 \mathrm{~mm}$. The temperature and the gas flow had a stimulating effect on the evaporation rate, while a local maximum of the evaporation rate was found as a function of the liquid thickness. A higher temperature causes a higher saturation pressure on the gas side of the liquid-gas interface, allowing more liquid to be evaporated at the same gas pressure. Moreover, the convection in the liquid phase is also better at a higher temperature. The gas flow rate has the same influence, albeit through another mechanism. A higher gas flow rate causes the gas boundary layer to decrease, causing a higher concentration gradient at the liquid-gas interface and thusly a higher evaporation rate. The local maximum of the evaporation rate as a function of the liquid thickness is explained as follows. On one hand, the confinement of the cover has an influence on the number of convection cells. It has been argued that the number of cells are such that the convection cells are neither too much compressed nor too dilated. On changing the liquid thickness, the aspect ratio changes as well, which forces the cells to get compressed or dilated. If the latter effect is too great, the number of cells changes. It appears that around the change of this number of cells, the critical curves of Marangoni convection find a maximum. This maximum appears to be in this work around liquid thicknesses of $2.5-3 \mathrm{~mm}$. This effect seems to be corroborated by the effect of the gas flow, which generates an overall convection. It seems that the maximum Marangoni counter flow velocities (triggered by the gas flow) also occurs around the same liquid thicknesses for which the evaporation rate is at its maximum. In conclusion, we can say that it is shown that the confinement principle, under the help of the gas flow, is responsible for the observed maximum of the evaporation rate as a function of the liquid thickness.

\section{Acknowledgements}

The authors acknowledge the financial support of the European Space Agency and of the Belgian Science Policy through the MAP Evaporation project and the Russian Science Foundation (Grant No. 15-19-20049 for the experimental part of the work).

\section{References}

Bénard, H., 1901. Les tourbillons cellulaires dans une nappe liquide transportant de la chaleur en régime permanent. Ann. Chem. Phys. 23, 62-144.

Bestehorn, M., Merkt, D., 2006. Regular surface patterns on Rayleigh-Taylor unstable evaporating films heated from below. Phys. Rev. Lett. 97, 127802.

Bestehorn, M., 2007. Convection in thick and in thin fluid layers with a free surface - The influence of evaporation. Eur. Phys. J.S.T. 146, 391-405.

Bodenschatz, E., Pesch, W., Ahlers, G., 2000. Recent developments in Rayleigh-Bénard convection. Annu. Rev. Fluid Mech. 32, 709-778.

Chandrasekhar, S., 1981. Hydrodynamic and hydromagnetic stability, second ed. Dover, New York.

Colinet, P., Legros, J.C., Velarde, M.G., 2001. Nonlinear Dynamics of surface-tensiondriven instabilities, first ed. Wiley-VCH Verlag, Berlin.

Dauby, P.C., Lebon, G., 1996. Bénard-Marangoni instability in rigid rectangular containers. J. Fluid Mech. 329, 25-64.

Dauby, P.C., Lebon, G., Bouhy, E., 1997. Linear Bénard-Marangoni instability in rigid circular containers. Phys. Rev. E 56, 520-530. 
Dauby, P.C., Colinet, P., Johnson, D., 2000. Theoretical analysis of a dynamic thermoconvective pattern in a circular container. Phys. Rev. E 61, 2663-2668.

Drazin, P., Reid, W.H., 1981. Hydrodynamic stability, second ed. Cambridge University Press, Cambridge.

Fang, G., Amirfazli, A., 2012. Understanding the edge effect in wetting: a thermodynamic approach. Langmuir 28, 9421-9430.

Getling, A.V., 1998. Rayleigh-Bénard convection: structures and dynamics, first ed. World Scientific, Singapore.

Gibbs, J.W., 1906. Scientific Papers, Longmans, London, p. 326 (Dover reprint, New York, 1961).

Grishaev, V., Amirfazli, A., Chikov, S., Lyulin, Y., Kabov, O., 2013. Study of edge effect to stop liquid spillage for microgravity application. Microgravity Sci. Technol. 1, 27-33.

Haut, B., Colinet, P., 2005. Surface-tension-driven instabilities of a pure liquid layer evaporating into an inert gas. J. Colloid Interface Sci. 285, 296-305 (2005).

Iorio, C. S., Goncharova, O., Kabov, O., 2011. Heat and mass transfer control by evaporative thermal pattering of thin liquid layers. Comput. Therm. Sci. 3, 333-342.

Li, Y. S., Liu, S.X., Yu, Y.X., Lai, G.Z., 1999. Numerical modeling of Boussinesq equations by finite element method. Coastal Eng. 37, 97-122.

Lyulin, Y., Kabov, O., 2014. Evaporative convection in a horizontal liquid layer under shear-stress gas flow. Int. J. Heat Mass Transfer 70, 599-609.

Machrafi, H., Rednikov, A., Colinet, P., Dauby, P.C., 2011. Bénard instabilities in a binaryliquid layer evaporating into an inert gas: Stability of quasi-stationary and time-dependent reference profiles. Eur. Phys. J.S.T. 192, 71-81.

Machrafi, H., Rednikov, A., Colinet, P., Dauby, P. C., 2013a. Time-dependent MarangoniBénard instability of an evaporating binary liquid layer including gas transients. Phys. Fluids 25, 084106.

Machrafi, H., Sadoun, N., Rednikov, A., Dehaeck, S., Dauby, P. C., Colinet, P., 2013b. Evaporation rates and Bénard-Marangoni supercriticality levels for liquid layers under an inert gas flow. Micrograv. Sci. Technol. 25, 251-265.

Machrafi, H., Iorio, C.S., Dauby, P.C., 2014. Relation between convective thermal patterns and heat flux through an evaporating surface via two-dimensional and three-dimensional numerical simulations. Interf. Phenom. Heat Transf. 2, 199-209.

Normand, C., Pomeau, Y., Velarde, M., 1977. Convective instability: a physicist's approach. Rev. Mod. Phys. 49, 581-624.

Pearson, J.R.A., 1958. On convection cells induced by surface tension. J. Fluid Mech. 4, 489-500. 